\title{
Urban land use mapping using high resolution SAR data based on density analysis and contextual information
}

\author{
Zhaohua Chen, Ying Zhang, Bert Guindon, Thomas Esch, Achim Roth, and Jiali Shang
}

\begin{abstract}
This paper presents a procedure for urban land use interpretation from a single high-resolution synthetic aperture radar (SAR) image. The approach involves two semi-automatic steps: urban extent delineation and urban land use mapping. In the first step, two general classes (urban and nonurban) are mapped using an existing method that involves analysis of speckle characteristics and intensity information. In the second step, more detailed urban land use classification is undertaken based on analysis of regional radar backscatter patterns in terms of density of dark linear features, density of bright features, and urban contextual information. Density analysis was conducted at three levels: individual building-road, urban block, and suburban commercial-industrial. Contextual information, including density, building size, and distance between buildings and parking places, was used to quantify urban morphological patterns. Tests were conducted for mapping Ottawa, Canada, using five Radarsat-2 images of different incidence angles and three TerraSAR-X images of the same incidence angles but different dates. The results show that the proposed method could be used to map five urban land uses including low-density residential, commercial-industrial, high-density urban, open land, and nonurban with accuracies in the range from $74 \%$ to $82 \%$.
\end{abstract}

\begin{abstract}
Résumé. Dans cet article, on présente une procédure pour l'interprétation de l'utilisation du sol en milieu urbain à partir d'une seule image radar à synthèse d'ouverture (RSO) haute résolution. L'approche comprend deux étapes semiautomatiques: la délimitation de l'étendue de l'espace urbain et la cartographie de l'utilisation du sol urbain. Dans la première étape, deux classes générales (urbain et non urbain) sont cartographiées à l'aide d'une méthode existante basée sur l'analyse des caractéristiques du chatoiement et de l'information sur l'intensité. Dans la deuxième étape, on effectue une classification plus détaillée de l'utilisation du sol urbain basé sur l'analyse des patrons régionaux de rétrodiffusion radar en termes de densité des caractéristiques linéaires sombres, de densité des caractéristiques claires et de l'information contextuelle urbaine. L'analyse de densité est réalisée selon trois niveaux de détails : édifice individuel/route individuelle, îlot urbain et zone commerciale/industrielle de banlieue. L'information contextuelle, incluant la densité, la taille des édifices et la distance entre les édifices et les places de stationnement, est utilisée pour quantifier les patrons de la morphologie urbaine. Des essais ont été réalisés pour cartographier la ville d'Ottawa, Canada, en utilisant cinq images Radarsat-2 à différents angles d'incidence et trois images TerraSAR-X ayant les mêmes angles d'incidence, mais acquises à des dates différentes. Les résultats montrent que la méthode proposée pourrait être utilisée pour cartographier cinq utilisations du sol urbain incluant le résidentiel à faible densité, les zones commerciales/industrielles, l'urbain à haute densité, les espaces libres et les espaces non urbains, avec des précisions variant de $74 \%$ à $82 \%$.

[Traduit par la Rédaction]
\end{abstract}

\section{Introduction}

Accurate mapping of urban environments and monitoring urban growth are important for urban development and environmental sustainability at the global level. The routine availability of synthetic aperture radar (SAR) data has rendered radar an option for timely urban environmental monitoring and map updating. However, interpretation of
SAR data of urban or semi-urban areas remains difficult due to geometric (foreshortening and lay-over) and radiometric (speckle and shadowing) characteristics of the imagery (Tupin and Roux, 2003). Urban areas are complex, being characterized by dense road networks, complicated distributions of buildings with different uses, as well as intervening open spaces of natural vegetation. Most urban pixels in remote sensing data contain contributions from

Received 7 July 2012. Accepted 12 December 2012. Published on the Web at http://pubs.casi.ca/journal/cjrs on 1 March 2013.

Zhaohua Chen, ${ }^{1}$ Ying Zhang, and Bert Guindon. Canada Centre for Remote Sensing, 588 Booth Street, Ottawa, ON, Canada K1A 0 Y7.

Thomas Esch and Achim Roth. German Remote Sensing Data Center, German Aerospace Center, Oberpfaffenhofen, 82234 Wessling, Germany.

Jiali Shang. Agriculture and Agri-Food Canada, 960 Carling Avenue, Ottawa, ON, Canada K1A 0C6.

${ }^{1}$ Corresponding author (e-mail: zhaohua.c@gmail.com). 
diverse covers (e.g., buildings, roads, grass, and trees), and the relative proportions of these components can vary widely within an urban pixel (Tupin et al., 2002). The backscattering from an urban area containing buildings can be complicated by the variation of orientation and structure of buildings and sensor geometry. Therefore, backscatter magnitude of urban areas in the SAR scene appears to have a wide range, and separating targets of interest (e.g., roads and buildings) from the background is very difficult if based solely on a threshold intensity value. In this regard, multitemporal, multifrequency, and polarized SAR data have proven to be useful in mapping land use classes (Pellizzeri et al., 2003; Haack, 2007; Li et al., 2010; Niu and Ban, 2012; Qi et al., 2012). The richness of texture in SAR images is also exploited to improve the differentiation of different land use classes (Dell'Acqua and Gamba, 2003; Pant et al., 2010). An enhanced separation of urban land use classes could be achieved by the combined optical and SAR data (Jiang et al., 2009; Ban et al., 2010; Zhu et al., 2011).

One main application arises in acquiring immediate information for rapid hazard response in urban regions hit by disasters such as earthquakes, flooding, or landslides. However, optical images are not always available depending on weather conditions. Multitemporal, multimode, and multipolarized SAR data have limitations when they are used for frequent monitoring and fast mapping because of the availability of the same orbit or mode (time requirement for multiple acquisitions by programming may not be practical for relief and rescue operations) and resolution (multipolarization or full polarimetric data are only available in reduced resolution). Even when available, the application of combined datasets from different modes or sensors can cause problems in registration and inconsistent backscattering characteristics. In terms of a fast and costefficient solution for the crisis situation, a single-polarized SAR may provide necessary information if processed properly. Efforts have been made to improve the accuracy of specific feature extraction from a single SAR scene, e.g., building detection (Stilla et al., 2003; Tison et al., 2007; Wegner et al., 2009; Poulain et al., 2011; Sportouche et al., 2011), and road detection (Tupin et al., 1998; Tupin et al., 2002; Dell'Acqua et al., 2003; Negri et al., 2006; Hedman et al., 2010). However, these feature extraction techniques are highly specialized, thus they are limited in their usefulness for operational mapping applications (Dell'Acqua et al., 2009).

With the launch of TerraSAR-X, COSMO-SkyMed, and Radarsat-2, very high-resolution SAR data are now available and sensitive enough to identify individual building characteristics and road networks. Thus there is a need to further explore the potential of radar for mapping more detailed land use classes and for quickly providing the needed information for the crisis situations. Recently, semiautomatic and fully automatic methods for mapping urban extent using a single-polarized scene of high-resolution SAR have been developed based on adaptive and iterative computation of local statistics (He et al., 2006), local autocorrelation and morphological processing (Stasolla and Gamba, 2008), and through analysis of local speckle divergence and intensity information (Esch et al., 2010). In the latter case, Esch et al. (2010) demonstrated that overall accuracies reached from $76 \%$ to $96 \%$ in mapping 12 urban areas around the world. However, these urban extent mapping approaches did not develop linkages between specific image features (e.g., lines, edges, etc.) to physical objects (e.g., buildings and road networks) and thus preclude refinement of interpretation to include urban sub-classes.

In the same vein, land use classification using traditional per-pixel classification methods based on statistics of intensity will be of limited value because pixels from different land cover types may have the same intensity values, or different parts of the same structure may exhibit differing levels of backscatter energy. For example, dark roofs and bright vegetation may look similar and residential areas may have the same brightness as commercial areas. Therefore, no approach based on per-pixel analysis is likely able to account for these subtleties (Stasolla and Gamba, 2008; Pamba and Aldrighi, 2011).

We argue, however, that analysis of regional radar backscatter patterns can be used to infer land use, as specific urban land uses may exhibit unique image spatial patterns of brightness. For example, attributes such as building size, building shape, building height, road network layout, and parking places are likely to differ among residential and commercial areas and between low and high intensity developments. However, areas of the same land use usually show similar spatial patterns. We argue that it is not necessary to accurately delineate relevant physical objects in any comprehensive way. Rather, if diagnostic image measures can be extracted (e.g., dark line segments being an indicator of the presence of roads) they can be used to infer land use in conjunction with other contextual information. We note that in the past, researchers have applied analogous principles to infer use from optical remote sensing data (Karathanassi et al., 2000; Zhang et al., 2002; Guindon and Zhang, 2009) and from SAR data (Tison et al. 2004; Niu and Ban, 2012).

Urban structural and contextual information from highresolution images can be analyzed at different scales (Dell'Acqua and Gamba, 2006; Guidon and Zhang, 2009; Pacifici et al., 2009). We view spatial characteristics of an urban area as consisting of individual elements (e.g., buildings, roads, and parking places), and grouping these elements into features on a larger scale according to land use functions. By grouping building, road, and parking place elements into blocks, we can then infer the land use of the block in question. We argue that it is the spatial scale of the grouping of structural features that distinguishes uses of interest (e.g., city block, rural area) (Guindon and Zhang, 2009). In SAR images, most buildings appear bright and can be distinguished from the background. However, roads, 
building shadows, and parking places all appear dark and are very hard to distinguish from each other. If bright appearances of buildings and dark appearances of roads, building shadows, and parking places are considered as inherent features of land use represented by SAR, then its distribution pattern will reflect the land use pattern. By analyzing the bright features (components of buildings) and dark linear features (composed of roads, building shadows, and parking places), land use characteristics can be described. The derived information on the density of houses and the orientation of the streets are very important for natural risk prevention or hazard mapping.

In this paper, we propose a semi-automated approach toward the urban land use mapping based on a single polarized SAR of very high resolution. The methodology first followed a technique presented in Esch et al. (2010) to map out the urban extent based on texture analysis. Then we performed detailed land use mapping based on density and contextual analysis of interested urban targets (building and road) at different scales.

\section{Study area and data preparation}

Tests were conducted for mapping Ottawa, Canada. Among the total urban land in the city of Ottawa, residential areas account for $37.3 \%$ of the city land area; transportation corridors occupy $21 \%$; institutional, commercial, and industrial areas occupy about $16.6 \%$, and recreation and open space about 20\% (City of Ottawa, 2006). The city of Ottawa is one of the fastest growing metropolitan areas in Canada. As the greenbelt in Ottawa restricts the expansion of the urban core area, urban sprawl occurred further away from the city center in major suburban areas in the west, south, and east end. Among these suburban areas exists a few planned communities with a low density of residential areas and a mix of densities of high technology industrial and commercial properties with large building sizes and parking spaces. These communities are also surrounded by large open spaces. Meanwhile buildings in downtown areas and some main thoroughfares are intensified with the high-rise blends of residential and commercial use due to high cost and limited space. Corresponding to the land use function and the density of the buildings and road networks in a city, a classification scheme that describes the urban sprawl containing low density residential areas, commercial areas, high-density areas, open land, and nonurban areas is adopted in this paper. Similar classes for describing urban land use based on density measures was also reported by Herold et al. (2002) in mapping Santa Barbara, California, using high-resolution digital aerial photographs.

To evaluate the robustness of our proposed methodologies, we conducted tests with a variety of radar sensors and imaging conditions, including both Radarsat-2 and TerraSAR-X. Our image data set includes five Radarsat-2 scenes of Spotlight mode with incidence angles from 24 to 48 degrees and three TerraSAR-X scenes acquired with the same incidence angles (39.9 to 42.8 degrees) but in different seasons (February, May, August) (Table 1). All data sets were acquired in $\mathrm{HH}$ polarization and subsequently orthorectified to a common projection and pixel spacing $(1.5 \mathrm{~m})$ using PCI Geomatica software. Our semi-automatic mapping procedure was implemented using ERDAS Imagine Modeler.

\section{Methodology}

Our urban mapping approach involves two semiautomatic steps, namely urban extent delineation and urban land use mapping. In the first step, two general classes (urban vs. nonurban) are mapped out by exploiting texture information from analyzing speckle characteristics and intensity information. The purpose is to roughly classify the urban scene to simplify procedures for a detailed map. In the second step, more detailed urban land use classification is conducted based on extraction of dark linear features and bright features through density and contextual analysis. Density of an attribute (e.g., linearity) is the proportion of pixels in a specified area of interest that exhibits an attribute response above a threshold. Density of a target feature usually does not share the same level of homogeneity at the same spatial scale. The urban regions can be extracted from a hierarchy of features under multiple scales. Density analysis is the process to determine the density of an attribute given an urban context. Contextual information including density, building size, and distance between buildings and parking places are used to describe urban morphological patterns.

Table 1. SAR Data used in this study.

\begin{tabular}{llcccc}
\hline Sensor & Beam & Date & Look-pass direction & Incidence angle (deg) & Resolution (m) \\
\hline Radarsat-2 & SLA6 & $2009-03-24$ & Right-desc & $34-35.3$ & $34.8-36.1$ \\
Radarsat-2 & SLA7 & $2010-01-30$ & Right-desc & $43.6-44.6$ & 2.7 \\
Radarsat-2 & SLA19 & $2010-01-29$ & Right-asc & $47.4-48.3$ & 2.2 \\
Radarsat-2 & SLA25 & $2010-01-27$ & Right-desc & $24-25.5$ & $39.9-42.28$ \\
Radarsat-2 & SLA74 & $2009-06-14$ & Right-desc & $39.9-42.28$ & 3.7 \\
TerraSAR-X & Strip012 & $2008-02-03$ & Right-desc & $39.9-42.28$ \\
TerraSAR-X & Strip012 & $2008-08-19$ & Right-desc & 3 \\
TerraSAR-X & Strip012 & $2009-05-21$ & Right-desc & 3 \\
\hline
\end{tabular}


In this study, density analysis was conducted at three levels of spatial scale; (i) individual buildings and road widths, (ii) urban block size, and (iii) suburban commercialindustrial block size. The scale for the feature extraction has to be large enough to cover the characteristic structures of the feature elements. The maximum dimension of the building and the maximum width of the road in the study area were used as a scale to measure the density of buildings and roads at the first level. The scale for measurement to characterize the land use pattern at the second or third level should contain a significant number of feature elements. Thus the scale of urban block was chosen to match the urban grid size, and the size of suburban commercialindustrial block was chosen to cover a typical commercial area. Threshold value of a target feature or a land use class was determined based on the statistics of known samples from ground observation and the minimum count number of the target that can be represented in the SAR according to its resolution. Figure 1 shows the principle processing steps.

\section{Delineation of urban extent}

The urban extent mapping adopts the pixel-based algorithm proposed in Esch et al. (2010). Assuming the noise in a SAR image of urban areas have a unique and high level of local speckle divergence $D_{x, y}$ (Figure 2a), the difference

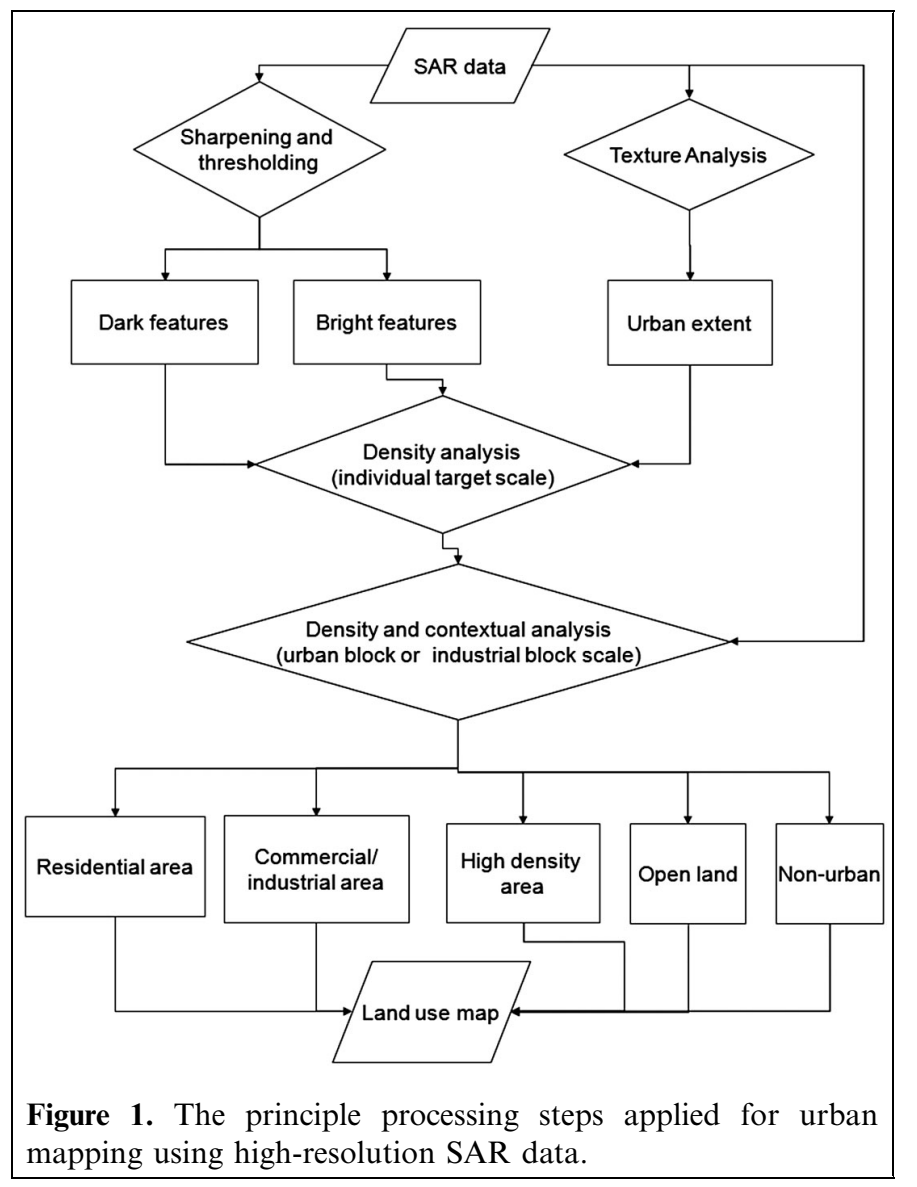

between the calculated local estimated coefficient of variation $C_{x, y}$ and the estimated scene-specific coefficient of variation $C$, are quantified by Esch et al. (2010) as

$D_{x, y}==C_{x, y}-C$ with $C_{x, y}=\frac{\sigma_{x, y}}{\mu_{x, y}}$

$C_{x, y}$ is defined by the local standard deviation $\sigma_{x, y}$, and the local mean $\mu_{x, y}$, is calculated via the maximumlikelihood estimators in a defined local neighbourhood. For a specific SAR scene, $C$ can be estimated based on the inverse of the equivalent number of looks, which is provided by the metadata file.

In the application of this paper, through texture analysis, $D_{x, y}, \mu_{x, y}$, and $\sigma_{x, y}$ were calculated and used as input for the urban extent classification procedure. By selecting thresholds corresponding to the mean and standard deviation texture measures, the urban extent is mapped. For more details about this algorithm, refer to Esch et al. (2010). After this semi-automated processing, two classes were mapped: urban and nonurban (Figure 2b). These two classes were used as basis for the following processing.

Due to the confusion of backscattering with other urban objects, water bodies may not be separated directly using this technique. In the case of calm conditions, water bodies in SAR images appear darker than other features and thus can be delineated through simple grey level thresholding. In this study, water areas were first detected using independent thresholds.

\section{Urban land use mapping}

\section{Extraction of preliminary dark and bright features}

To exploit contextual information for land use mapping, we first located SAR image features that exhibit spatioradiometric attributes expected from reflection from buildings, roads, and parking places. For example, we anticipated that buildings, bridges, and large foliated trees should appear bright, the first two because of double and multiple bounce effects and the latter due to "roughness" effects on the scale of the SAR sensor wavelength. On the other hand, smooth surfaces such as roads, parking places, and herbaceous vegetated surfaces should appear dark due to specular reflection. These assumptions are in agreement with similar observations made by other researchers, e.g., that bright targets in urban scenes have been often considered as indicative of man-made raised structures (Stasolla and Gamba, 2008; Esch et al., 2010). In addition to radiometry, roads should appear as dark line-like features. We therefore assumed that the presence and distribution of bright features in the SAR scene is indicative of the presence and pattern of buildings in the real world in urban area. The same assumption was applied to the dark linear features for roads. Therefore, our subsequent analysis involved finding and characterizing bright features and dark linear features. Preliminary extraction of bright features and dark features 


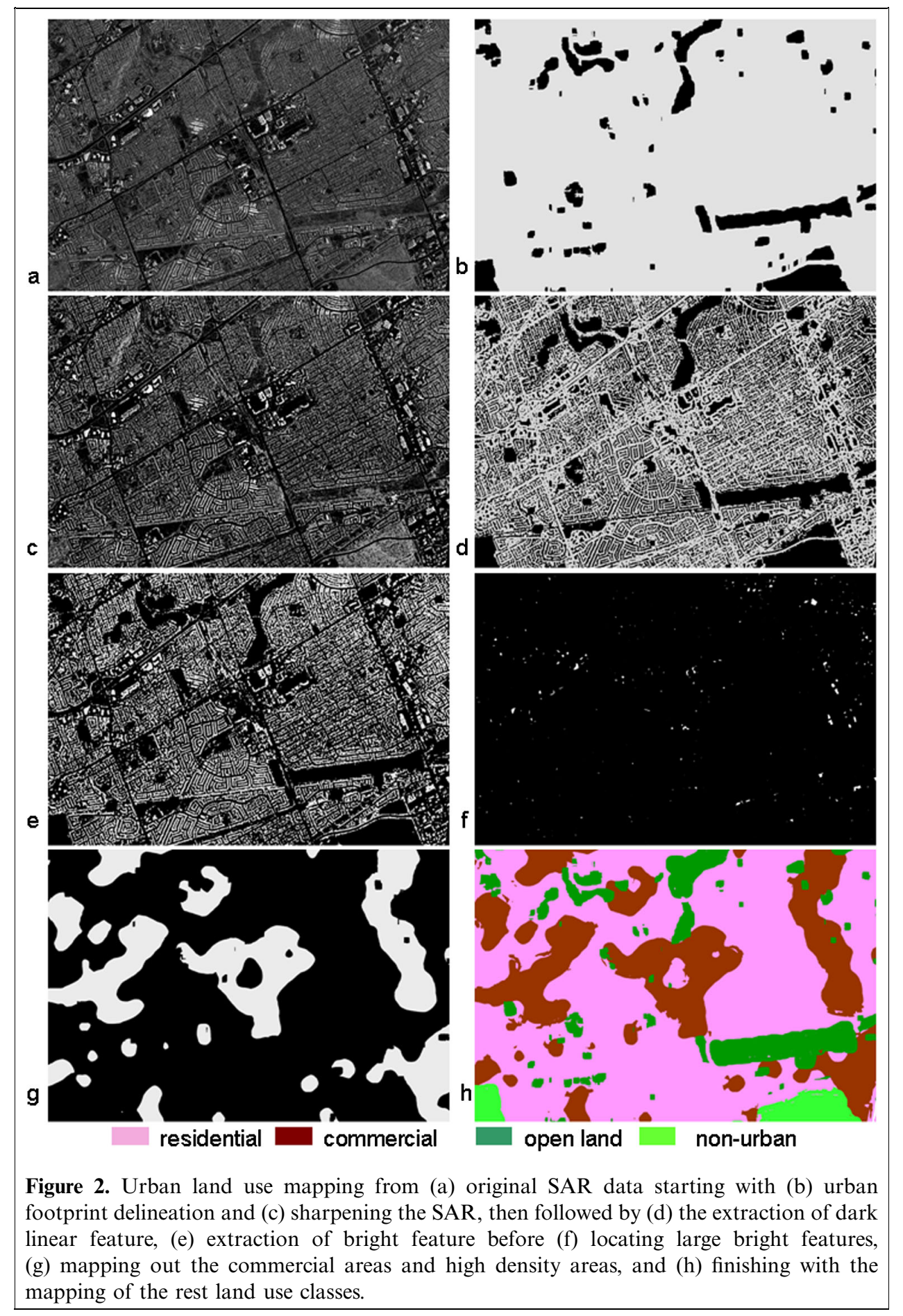

from the background can be based on intensity. However, due to the existence of speckle, man-made structures (e.g. roads, buildings, parking places) and natural objects (e.g., trees and grass) were indistinguishable.

Speckle filtering and sharpening techniques were used to improve both the definition of individual targets of interest and their local patterns. The SAR image was first smoothed with a median filter of 9 pixels $\times 9$ pixels. A sharpened image was acquired by applying a mean filter with a window size of 33 pixels $\times 33$ pixels to the median filtered image first, then adding the difference between the mean filtered image and the median filtered image back to the median filtered image. As a result of this pre-processing, each individual element (e.g., building or road segment) was more easily distinguished from the background than the original image (Figures 2c and 3). For example, pixels from a single building may have variations in brightness in original SAR image, thus some parts of the building appear dark and others bright. After this pre-processing, most pixels from a single building appeared brighter than the surroundings. 
Figure 3 shows that the buildings and most roads are distinguishable from the background in the processed image, and a few regional backscatter patterns clearly show the characteristics of land uses. For example, a residential area shows the consistent linear distributions of small bright features and dark linear features (Figure 3a); a commercialindustrial area shows the distribution of large dark features between large bright features (Figures 3b and 3c); and a high urban density area is characterized by clustering of large bright features with scattered dark features.

The maximum intensity value of the sample area of known road segments was used as a threshold to separate bright from dark features on the sharpened image. Due to insufficient illumination and contrast in the image, it was difficult to separate buildings from nearby trees and street segments from grass or building shadows, and the detected buildings and streets sometimes only showed incomplete shapes or sizes. As a result, some tree areas were included in the bright feature overlay, while some low vegetation areas were included among dark features. The unwanted information will be removed in the following refining procedures.

\section{Refining of dark and bright features through density analysis at a scale of individual target size}

On dark features, we focused on extracting dark linear features that were directly linked to road network and large dark features that were linked to parking places. On bright features, we further separated the large bright features that were linked to large building and nonbuilding areas. The refining process is based on the density analysis of dark features and bright features within a given window of a size equal to the maximum two dimensions of the target (road segment or building) in the study area. This procedure produces a local texture feature characterizing the properties in small regions centered on sample positions. The advantage of this window selection is that each component of the local feature defines an image, which, as such, can be displayed and processed using conventional methods.

In this study, the maximum width of a 35 pixel $(50 \mathrm{~m})$ dark linear feature was found to characterize the highway in the SAR scene, thus applying a window size of 35 pixels $\times$ 35 pixels to the dark features; density was calculated and used to extract the dark linear features. Although $50 \mathrm{~m}$ is much wider than typical roads, it was found to be an effective scale to describe the existence of road segments from the SAR image. Any SAR pixels with an assigned density value less than $100 \%$ but more than $24.5 \%$ of the total pixels within the window (the threshold is determined from the known road samples) were considered as linear feature candidates. Then by overlaying with the urban extent layer, sieving out the linear feature candidate that is isolated and with small area coverage (less than $180 \mathrm{~m}^{2}$ in size), dark linear features were mapped out (Figure 2d). By doing this, dark features of large size (parking lots, large vegetated areas, and bare surfaces) and isolated features (small open space, tree shadows, and building shadows) were masked out. Only roads and parts of long features including building shadows, linear parking lots, and open areas between neighbouring buildings or along roads were kept as linear features. This dark linear feature layer was used for linear feature density analysis at block unit. The dark

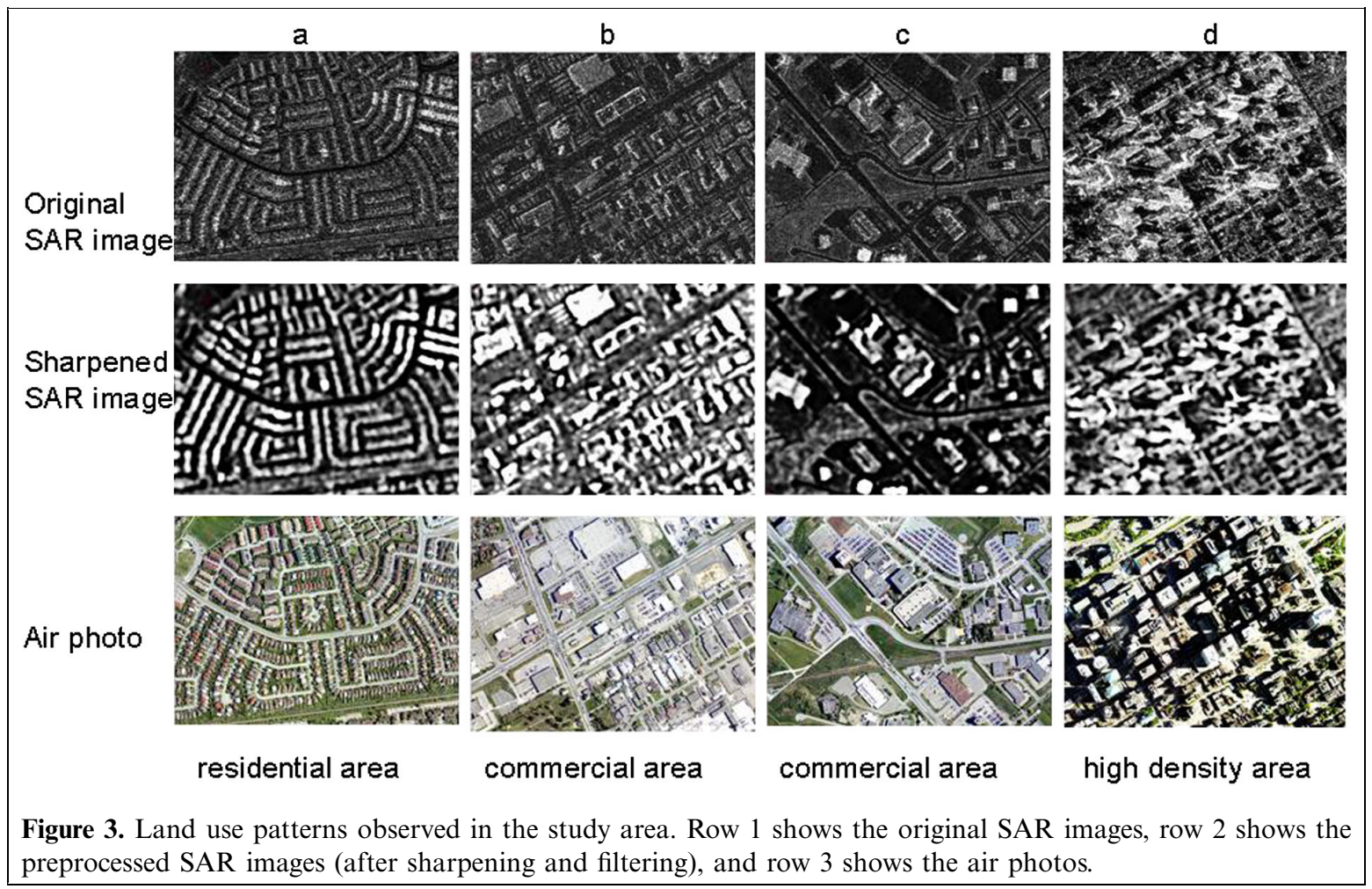


features with large density $(100 \%$ covered within the window) were kept as large dark feature layer (e.g., parking lots, large vegetated areas, and bare surfaces) for the further processing to derive the potential large parking places.

When applying the density analysis on bright features at a window size of $25 \times 25$ (maximum residential house width in the scene), if the density value of a pixel was more than $5 \%$ of the window the pixel was kept as part of the bright features (Figure 2e), otherwise it was labelled as a nonbuilding. This was to remove the trees and cars that have similar radiometric characteristics as buildings but smaller spatial sizes. Considering that the actual width (shorter side) of a regular house is smaller than the commercial buildings, analysis on the bright features at the individual building width (maximum house width in the scene) will separate the features representing regular residential buildings from those of large buildings. If the density value of a pixel was greater than $80 \%$ of the total pixels within the window (i.e., individual building size over $1250 \mathrm{~m}^{2}$ ) and located within an urban area mapped previously, the pixel was labelled as a large bright feature (Figure 2f). After this step, a binary layer of large bright feature and a binary layer of nonbuildings were created.

\section{Mapping out land use classes based on further density and contextual analysis}

Based on the extracted features, information about the potential location and distribution pattern of buildings and streets can be inferred. Assuming the extracted information about the potential location and distribution of buildings and roads can still generally reflect urban morphological characteristics, we could then conduct the land use classification. In urban areas, road, parking places, and buildings coexist naturally and form certain patterns depending on the use of the building for residential areas or commercial areas. Once these features were separated, the urban morphological pattern was identified. Two scales were used for land use mapping, including urban block size, and suburban commercial-industrial block size. Residential areas and high density areas were mapped at urban block size of $200 \mathrm{~m} \times 200 \mathrm{~m}$ (a window size of 135 pixels $\times 135$ pixels). In many North American cities, urban development has followed specific land use zoning. As a result, many commercial and industrial buildings can be found clustering in a suburban block bigger than a regular residential block. In this study, commercial areas and industrial areas were mapped at a suburban commercial-industrial block size of $300 \mathrm{~m} \times 300 \mathrm{~m}(201$ pixels $\times 201$ pixels $)$.

Suburban commercial-industrial areas exhibited two spatial patterns in the study area. The first pattern involved large buildings surrounded by parking lots that were usually of urban block size and adjacent to residential areas. This type typically comprised large shopping centres, gas stations, office buildings, and institutional buildings. The second pattern occurred on a larger spatial scale and comprised mixes of large and small buildings surrounded by parking infrastructure. Examples of this pattern type were industrial plants and warehouses in suburban industrial parks. In summary, commercial-industrial areas need to be analyzed at two mapping scales. The principal steps in commercial-industrial area mapping based on density analysis and contextual information are described below.

First, density analysis at the urban block scale was conducted to identify and discard nonbuilt-up areas. If an area at this scale exhibited a dark linear feature density less than $11 \%$ (based on the statistics from a typical urban block, discussed later) and was part of the nonbuilding layer, it was labelled as a nonbuilt-up candidate. Overlaying the nonbuilt-up candidate layer with the large dark feature layer, we derived a layer of potential parking areas. By subtracting the potential parking places from nonbuilt-up candidates, we generated a nonbuilt-up layer; then a built-up layer was created that included all the area that was not included in the nonbuilt-up area. The information from this density analysis for built-up and nonbuilt-up areas was complementary to the previously mapped urban extent for urban and nonurban area.

Second, commercial-industrial core mapping was done. When calculating the distance to large bright features and potential parking areas, if the areas were within $45 \mathrm{~m}$ to the large bright feature or within $45 \mathrm{~m}$ from the large bright feature to the potential parking area, they were considered as commercial-industrial core area.

Third, commercial area mapping at the industrial-rural block scale was done. When calculating the density of commercial core pixels at the industrial-rural unit, if the pixel had a density value in the commercial core area of more than $45 \%$ of the unit area and was not located in a nonbuilt-up area, it was then considered as a commercialindustrial area (Figure 2g).

From the SAR, high-rise buildings in the dense urban core appear very bright due to various corner and doublebouncing reflections from the building walls, structures at the rooftop, and neighbouring buildings. This makes it possible to map out the high density urban areas in SAR. In this study, high density areas were only mapped at block scale following two steps. First, an appropriate intensity threshold was selected, based on known sample areas in median filtered SAR, and the pixels were retained with intensity values in excess of the threshold (so-called bright reflectors). Second, the density of the bright reflectors was calculated resulting in the density of the large bright feature at block size. A threshold for the density of the bright reflectors and a threshold for the density of large bright feature was selected based on the known sample areas, and the pixels that exceeded the thresholds set for two layers were retained. This procedure results in the high-density urban area.

A low-density residential area includes single homes, townhomes, and scattered multistory apartment buildings. Once the built-up areas, the low-density commercial-industrial 
areas and the high-density urban areas were mapped, the low-density residential area was mapped by subtracting the low-density commercial-industrial areas and the high-density urban areas from the built-up areas.

Open area refers to an area that has no man-made structures and includes parks and vegetated area within a city. Selecting a threshold value for the size of a nonbuilt-up area in the nonbuilt-up layer, open areas were mapped as nonbuilt-up areas with a smaller size than the threshold value, and were surrounded by an urban area (based on the calculation of distance to an urban area). Nonurban area was the area with a size exceeding the threshold value.

When five individual classes were generated using the outlined procedures, these classes and the water class were then merged to generate a final land use map (Figure 2h).

\section{Results and discussion}

The proposed method was applied to the eight $\mathrm{HH}$ polarized SAR scenes listed in Table 1. Although these images were acquired from 2008-2010, we evaluated our methods on urban test areas that did not undergo significant change during this period. As a result, performance results for the eight scenes can be compared with each other. The large water body (Ottawa River) in the study area has variations of brightness in the SAR images depending on the seasons. It is hard to separate a snow and ice-covered water area from other urban features due to similar brightness in a SAR scene acquired in winter. Water area has the lowest brightness value in a SAR scene acquired in summer. However, the wind and waves may affect the local variations of backscattering of the water. Thus two scenes of TerraSAR-X images acquired in the summer were used to make a complete water mask, and the derived water class was not evaluated for its accuracy.

The mapping accuracy for five land use classes including residential, commercial-industrial, high-density areas, open land, and nonurban areas were evaluated using a stratified random sampling strategy. The test samples were collected across each scene. Due to the difference in coverage between TerraSAR-X and Radarsat-2 scenes, a different number of random samples were used. For three TerraSAR-X scenes, 690 random pixels were selected to evaluate the mapping accuracy because of the same coverage. For Radarsat-2 data, 309 random samples for SLA6, 335 for SL7, 420 for SLA19, 348 for SLA25, and 387 for SLA74 were used. The classification categories of these samples were made based on visual interpretation of airphotos, Google maps, and ground visits. Table 2 shows the mapping results. As the Radarsat-2 scenes do not cover the high-density urban area that is located in the downtown area of the capital region, there is no high-density urban area detected in five Radarsat-2 scenes. One thing to note about the land use mapping procedure proposed in this study is that it was based on accurate mapping of urban extent. It was found that nonurban area achieved the highest mapping accuracy among all five classes, with producer accuracy ranging from $86 \%$ to $100 \%$ and user accuracy ranging from $75 \%$ to $91 \%$. Open land class achieved the lowest mapping accuracy, with producer accuracy ranging from $24 \%$ to $60 \%$ and user accuracy ranging from $79 \%$ to $100 \%$. Low accuracy of open areas was due to the application of a large size window for texture analysis during the urban extent mapping, during which, more urban areas were mapped at the sacrifice of the open areas among the built-up areas. In general, higher accuracy was achieved for the residential class than the commercial-industrial class. Within commercial-industrial areas, the areas around large buildings were mapped successfully; however, areas around small buildings could not be mapped efficiently because of the lack of consideration of the contextual information for mapping commercial-industrial areas with small buildings. As highdensity areas only exist in a limited area in the study area, the results showed producer accuracy ranging from $47 \%$ to $65 \%$ and user accuracy ranging from $80 \%$ to $100 \%$.

In this study, we found that contextual information is very useful in improving urban land use classification. The combination of bright feature and dark linear feature density is useful to correctly characterize the urban features. We found that the dark linear feature density and bright feature density in the urban area in a typical block is greater than $11 \%$, which is selected as threshold to determine if the

Table 2. Results of urban land use mapping using high-resolution SAR based on analysis of linear feature and building density and contextual information.

\begin{tabular}{|c|c|c|c|c|c|c|c|c|c|c|c|c|}
\hline \multirow[b]{2}{*}{ Scene } & \multicolumn{5}{|c|}{ Producer's accuracy $(\%)$} & \multicolumn{5}{|c|}{ User's accuracy (\%) } & \multirow[b]{2}{*}{$\begin{array}{c}\text { Overall } \\
(\%)\end{array}$} & \multirow[b]{2}{*}{ Kappa } \\
\hline & Residential & Commercial & $\begin{array}{c}\text { High } \\
\text { density }\end{array}$ & $\begin{array}{c}\text { Open } \\
\text { land }\end{array}$ & Nonurban & Residential & Commercial & $\begin{array}{c}\text { High } \\
\text { density }\end{array}$ & $\begin{array}{l}\text { Open } \\
\text { land }\end{array}$ & Nonurban & & \\
\hline TSX 20080203 & 88.6 & 60.9 & 64.7 & 59.4 & 95.2 & 78.5 & 67.9 & 100.0 & 90.1 & 85.3 & 81.3 & 0.74 \\
\hline TSX 20080819 & 82.0 & 63.2 & 47.1 & 52.8 & 99.0 & 82.3 & 65.5 & 80.0 & 97.0 & 74.9 & 78.7 & 0.7 \\
\hline TSX 20090521 & 83.1 & 56.3 & 64.7 & 49.6 & 98.6 & 78.8 & 63.6 & 84.6 & 95.3 & 76.8 & 78.0 & 0.69 \\
\hline RS2 20090324 & 86.3 & 51.4 & na & 24.3 & 85.7 & 58.6 & 67.9 & na & 100.0 & 90.9 & 74.4 & 0.61 \\
\hline RS2 20100130 & 74.8 & 89.8 & na & 68.8 & 95.8 & 84.1 & 73.3 & na & 88.0 & 81.3 & 81.8 & 0.75 \\
\hline RS2 20100129 & 85.7 & 91.5 & na & 29.9 & 95.2 & 82.2 & 69.1 & na & 88.5 & 76.7 & 78.1 & 0.69 \\
\hline RS2 20100127 & 86.3 & 65.9 & na & 36.5 & 87.1 & 75.0 & 67.5 & na & 79.2 & 82.1 & 76.7 & 0.66 \\
\hline RS2 20090614 & 88.1 & 51.0 & na & 50.8 & 100.0 & 82.9 & 92.9 & na & 91.4 & 75.6 & 80.9 & 0.72 \\
\hline
\end{tabular}


area is built-up or nonbuilt-up. However, the ranges of linear feature density and bright feature density in residential areas, commercial-industrial areas, and high-density areas have overlaps, thus a single threshold value could not be selected to determine the land use type (Figure 4).

As we can see in Figure 3 (air photo) and Figure 5a, in residential areas buildings and roads are distributed with relatively uniform size and space. Bright lines that represent extracted bright features (mostly buildings) and linear features (mostly roads) occur at a regular interval. However, commercial-industrial areas are characterised with buildings and parking spaces of different sizes. The extracted bright features and dark linear features in commercial-industrial areas do not occur at regular intervals like that in residential areas. Extracted linear features close to big buildings also include large parts of building shadows and parking places (Figures 5b and 5c). In high-density urban areas, high-rise buildings have a larger size than residential areas and roads usually distribute regularly. However, the extracted shapes of high-rise buildings and roads are irregular and incomplete due to the layover and shadow effect (Figure 5d). Therefore, in this study, we applied contextual information in addition to the density information.

Density analysis is based on the accurate mapping of road and building candidates that depend on the geometric and sensor characteristics. Although the brightness of the same object in five Radarsat-2 images is different due to different incidence angles and look directions, the results suggest that variation of incidence angles may not produce much variation in mapping accuracy. This may be due to the procedures applied for mapping ensured extraction of consistent density information and contextual information. However, more experiments need to be done to verify the influence because scenes that were used in this study have different coverage. Two scenes acquired in winter (RS2 of 20100130 and TSX of 20080203) produced better results than those from summer because trees were distinguished from buildings in winter.

The extraction of water bodies using threshold value may only work for large and quiet water bodies, not for small water bodies or large water bodies with torrents. Other efficient algorithms may be studied for further application. It was found that the extracted boundaries between the different regions were not clear due to the use of a large window size for describing land uses. It was also noted that where the cardinal effects are pronounced in the scene, identical objects may have different backscattering depending on their orientations. Thus, identification of targets in those areas remains problematic. In future, the algorithms used for building detection and linear feature detection may be substituted by the methods proposed by other researchers to achieve better overall results. Improvements for maintaining the boundaries of different land uses should be considered. Regularity of linear features may also be explored as contextual information during the classification.

The window size and threshold value for mapping the urban elements are set manually according to the knowledge of the study area and SAR scene characteristics. This can be one of the limitations of the proposed method when it is used for other study areas. Scales of density measurement,

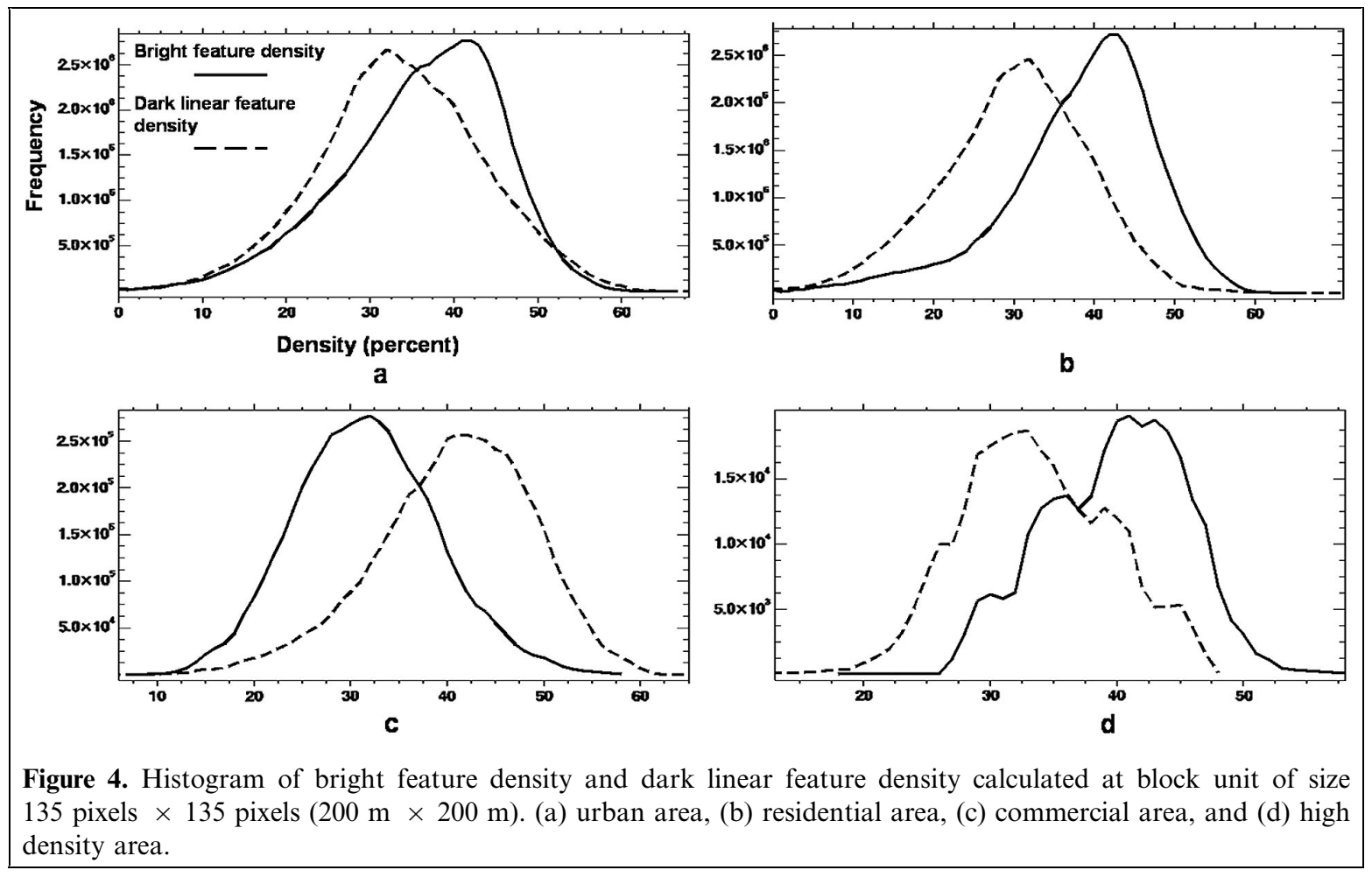




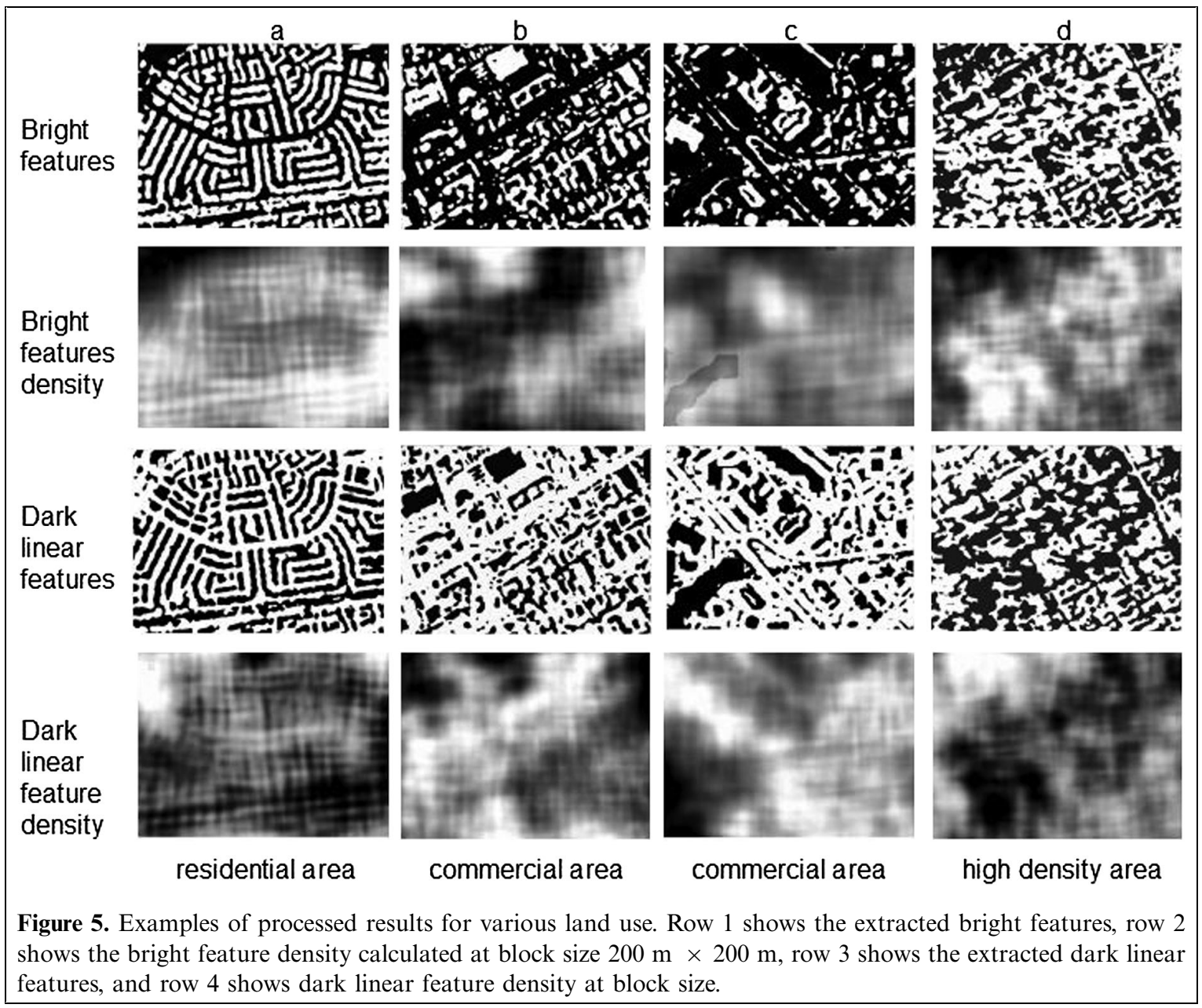

size, and shape of urban forms may vary from one city to another. The scales adopted in this study may not be suitable for another city and may need to be adjusted to suit the situation of the mapping area during the application. The semi-automatic procedure allows the user to adjust only a few parameters according to the scene at the beginning of the data processing. It would speed up the processing of large volumes of SAR data of the same area. A dark linear feature layer and a bright feature layer that were created during the mapping procedures may be used for monitoring purposes and verification purposes.

\section{Conclusion}

Urban land use mapping using a single SAR scene has been a challenge. A new semi-automatic procedure for urban land use mapping based on analysis of regional radar backscatter patterns in terms of density of dark linear features, bright features, and contextual information was developed and presented in this paper. The method was tested using the high-resolution data from Radarsat- 2 and TerraSAR-X sensors for land use mapping at the city level. Applying this method, urban land use mapping can achieve up to $74-82 \%$ overall accuracy for five land use classes including low-density residential, low-density commercialindustrial, high-density urban areas, open land, and nonurban ares. The results from this study suggest that the knowledge of land use could be acquired from the contextual information by analysis of radar backscatter patterns at three levels of details. By properly selecting the measurement levels for density, the land use pattern can be revealed. The developed method is complementary to existing methods for urban extent mapping.

The research shows that the method achieves higher accuracy for the nonurban class but lower accuracy for the open land class and needs more consideration. The test with data from different incidence angles suggests that the method may be used for mapping and monitoring, thus allowing the exploitation of various SAR data sets from Radarsat- 2 and TerraSAR-X that provide images with a wide range of incidence angles.

\section{Acknowledgements}

The authors would like to thank Mr. Achim Roth and Dr. Thomas Esch from the German Aerospace Center (DLR) for providing the scripts for urban mask mapping and 
TerraSAR-X data for this paper; MacDonald, Dettwiler, and Associates Ltd. for providing Radarsat-2 data; and Pierre Sauve from the Geodetic Survey Division, Natural Resources Canada for providing ground control points.

\section{References}

Ban, Y., Hu, H., and Rangel, I.M. 2010. Fusion of Quickbird MS and RADARSAT SARfor urban land-cover mapping: object-based and knowledge-based approach. International Journal of Remote Sensing, Vol. 31, No. 6, pp. 1391-1410. doi: 10.1080/01431160903475415.

City of Ottawa, 2006. 2005 Land use survey. Publication \#13-24.

Dell'Acqua, F., and Gamba, P. 2006. Discriminating urban environments using multiscale texture and multiple SAR images. International Journal of Remote Sensing, Vol. 27, No. 18, pp. 3797-3812. doi: 10.1080/0143 1160600557572.

Dell'Acqua, F., Gamba, P., and Lisini, G. 2003. Road map extraction by multiple detectors in fine spatial resolution SAR data. Canadian Journal of Remote Sensing, Vol. 29, No. 4, pp. 481-490. doi: 10.5589/m03-019.

Dell'Acqua, F., and Gamba, P. 2003. Texture-based characterization of urban environments on satellite SAR images. IEEE Transactions on Geoscience and Remote Sensing, Vol. 41, No. 1, pp. 153-159. doi: 10.1109/TGRS.2002.807754

Dell'Acqua, F., Gamba, P., and Lisini, G. 2009. Rapid mapping of high resolution SAR scenes. ISPRS Journal of Photogrammetry and Remote Sensing, Vol. 64, No. 5, pp. 482-489. doi: 10.1016/j.isprsjprs. 2008. 09.006 .

Esch, T., Thiel, M., Schenk, A., Roth, A., Muller, A., and Dech, S. 2010. Delineation of urban footprints from TerraSAR-X data by analyzing speckle characteristics and intensity information. IEEE Transactions on Geoscience and Remote Sensing, Vol. 48, No. 2, pp. 905-916. doi: 10.1109/TGRS.2009.2037144.

Guindon, B., and Zhang, Y. 2009. Automated urban delineation from Landsat imagery based on spatial information processing. Photogrammetric Engineering \& Remote Sensing, Vol. 75, No. 7, pp. 845-858.

Haack, B. 2007. A comparison of land use/cover mapping with varied radar incident angles and seasons. GIScience \& Remote Sensing, Vol. 44, No. 4, pp. 305-319. doi: 10.2747/1548-1603.44.4.305.

He, C., Xia, G., and Sun, H. 2006. An adaptive and iterative method of urban area extraction from SAR images. IEEE Transactions on Geoscience and Remote Sensing, Vol. 3, No. 4, pp. 504-507. doi: 10.1109/ LGRS.2006.878447.

Hedman, K., Stilla, U., Lisini, G., and Gamba, P. 2010. Road network extraction in VHR SAR images of urban and suburban areas by means of class-aided feature-level fusion. IEEE Transactions on Geoscience and Remote Sensing, Vol. 48, No. 3, pp. 1294-1296. doi: 10.1109/TGRS. 2009.2025123.

Herold, M., Scepan, J., and Clarke, K.C. 2002. The use of remote sensing and landscape metrics to describe structures and changes in urban land uses. Environment and Planning A, Vol. 34, pp. 1443-1458. doi: 10.1068/ a3496.

Karathanassi, V., Iossifidis, C., and Rokos, D. 2000. A texture based classification method for classifying built areas according to their density. International Journal of Remote Sensing, Vol. 21, No. 9, pp. 1807-1823. doi: 10.1080/014311600209751.
Li, X., Pottier, E., Guo, H., and Ferro-Famil, L. 2010. Urban land cover classification with high-resolution polarimetric SAR interferometric data. Canadian Journal of Remote Sensing, Vol. 36, No. 3, pp. 236247. doi: $10.5589 / \mathrm{m} 10-046$.

Negri, M., Gamba, P., Lisini, G., and Tupin, F. 2006. Junction-aware extraction and regularization of urban road networks in high-resolution SAR images. IEEE Transactions on Geoscience and Remote Sensing, Vol. 44, No. 10, pp. 2962-2971. doi: 10.1109/TGRS.2006.877289.

Niu, X., and Ban, Y. 2012. An adaptive contextual SEM algorithm for urban land cover mapping using multitemporal high-resolution polarimetric SAR data. IEEE Journal of Selected Topics in Applied Earth Observations and Remote Sensing, Vol. 5, No. 4, pp. 1129-1139. doi: 10.1109/JSTARS.2012.2201448

Pacifici, F., Chini, M., and Emery, W.J. 2009. A neural network approach using multi-scale textural metrics from very high resolution panchromatic imagery for urban land-use classification. Remote Sensing of Environment, doi: 10.1016/j.rse.2009.02.014

Pamba, P., and Aldrighi, M. 2011. SAR-based analysis of urban areas: Joint exploitation of segments and statistical features. In Proceedings: IEEE GRSS and ISPRS Joint Urban Remote Sensing Event (JURSE), April 11-13, 2011, Munich, Germany. pp. 169-172.

Pant, T., Singh, D., and Srivastava, T. 2010. Advanced fractal approach for unsupervised classification of SAR images. Advances in Space Research, Vol. 45, No. 11, pp. 1338-1349. doi: 10.1016/j.asr.2010.01.008.

Pellizzeri, T.M., Gamba, P., Lombardo, P., and Dell'Acqua, F. 2003. Multitemporal/multiband SAR classification of urban areas using spatial analysis: statistical versusneural kernel-based approach. IEEE Transaction on Geoscience and Remote Sensing, Vol. 41, No. 10, pp. 2338-2353. doi: 10.1109/TGRS.2003.818762.

Poulain, V., Inglada, J., Spigai, M., Tourneret, J., and Marthon, P. 2011. High-resolution optical and SAR image fusion for building database updating. IEEE Transactions on Geoscience and Remote Sensing, Vol. 49, No. 8, pp. 2900-2910. doi: 10.1109/TGRS.2011.2113351.

Qi, Z., Yeh, A., Li, X., and Lin, Z. 2012. A novel algorithm for land use and land cover classification using RADARSAT-2 polarimetric SAR data. Remote Sensing of Environment, Vol. 118, pp. 21-39. doi: 10.1016/j.rse. 2011.11.001.

Sportouche, H., Tupin, F., and Denise, L. 2011. Extraction and threedimensional reconstruction of isolated buildings in urban scenes from high-resolution optical and SAR spaceborne images. IEEE Transactions on Geoscience and Remote Sensing, Vol. 49, No. 10, pp. 3932-3946. doi: 10.1109/TGRS.2011.2132727.

Stasolla, M., and Gamba, P. 2008. Spatial indexes for the extraction of formal and informal human settlements from high resolution SAR images. IEEE Journal of Special Topics on Applications of Remote Sensing, Vol. 1, No. 2, pp. 98-106. doi: 10.1109/JSTARS.2008.921099.

Tison, C., Nicolas, J.M., Tupin, F., and Maitre, H. 2004. A new statistical model for Markovian classification of urban areas in high-resolution SAR images. IEEE Transactions on Geoscience and Remote Sensing, Vol. 42, No. 10, pp. 2046-2057. doi: 10.1109/TGRS.2004.834630.

Tison, C., Tupin, F., and Maitre, H. 2007. A fusion scheme for joint retrieval of urban height map and classification from high-resolution interferometric SAR images. IEEE Transactions on Geoscience and Remote Sensing, Vol. 45, No. 2, pp. 496-505. doi: 10.1109/TGRS.2006. 887006.

Tupin, F., Houshmand, B., and Datcu, M. 2002. Road detection in dense urban areas using SAR imagery and the usefulness of multiple views. 
IEEE Transactions on Geoscience and Remote Sensing, Vol. 40, No. 11, pp. 2405-2414. doi: 10.1109/TGRS.2002.803732.

Tupin, F., Maitre, H., Mangin, J.F., Nicolas, J.M., and Pechersky, E. 1998. Detection of linear features in SAR images: application to road network extraction. IEEE Transactions on Geoscience and Remote Sensing, Vol. 36, No. 2, pp. 434-453. doi: 10.1109/36.662728.

Tupin, F., and Roux, M. 2003. Detection of building outlines based on the fusion of SAR and optical features. ISPRS Journal of Photogrammetry and Remote Sensing, Vol. 58, No. 1-2, pp. 71-82. doi: 10.1016/S09242716(03)00018-2.

Wegner, J.D., Thiele, A., and Soergel, U. 2009. Fusion of optical and InSAR features for building recognition in urban areas. In: The
International Archives of the Photogrammetry, Remote Sensing and Spatial Information Sciences, Vol. XXXVIII, Part 3/W4, pp. 169-174.

Zhang, Q., Wang, J., Peng, X., Gong, P., and Shi, P. 2002. Urban built-up land change detection with road density and spectral information from multi-temporal Landsat TM data. International Journal of Remote Sensing, Vol. 23, No. 15, pp. 3057-3078. doi: 10.1080/014311601101 04728 .

Zhu, Z., Woodcock, C., Rogan, J., and Kellndorfer, J. 2011. Assessment of spectral, polarimetric, temporal, and spatial dimensions for urban and peri-urban land cover classification using Landsat and SAR data. Remote Sensing of Environment, Vol. 117, pp. 72-82. doi: 10.1016/j.rse. 2011.07.020. 